\title{
Inverted urothelial papilloma of the upper urinary tract: description of two cases with systematic literature review
}

\author{
R. Santi ${ }^{1}$, I. C. Galli ${ }^{2}$, V. Canzonieri ${ }^{3,4}$, J. I. Lopez ${ }^{5}$ and G. Nesi ${ }^{*}$
}

\begin{abstract}
Background: Inverted urothelial papilloma (IUP) of the upper urinary tract is an uncommon benign tumour that occasionally presents as a polypoid mass causing urinary obstruction. Histologically, IUP is characterised by a proliferating urothelium arranged in cords and trabeculae, in continuity with overlying intact epithelium, and extending into the lamina propria in a non-invasive, endophytic manner. Cytological atypia is minimal or absent. Top differential diagnoses include urothelial carcinoma with inverted growth pattern and florid ureteritis cystica. Although urothelial carcinomas of the upper urinary tract with prominent inverted growth pattern commonly harbour microsatellite instability, the role of the mutator phenotype pathway in IUP development is still unclear. The aim of this study was to describe two additional cases of IUP of the upper urinary tract, along with an extensive literature review.
\end{abstract}

Case presentation: We observed two polypoid tumours originating in the renal pelvis and the distal ureter, respectively. Both patients, a 76-year-old woman and a 56-year-old man, underwent surgery because of the increased likelihood of malignancy. Histology was consistent with IUP and patients are alive and asymptomatic after long-term follow-up ( 6 years for the renal pelvis lesion and 5 years for the ureter lesion). The tumours retained the expression of the mismatch-repair protein MLH1, MSH2, and PMS2 whereas loss of MSH6 was found in both cases.

Conclusions: When completely resected, IUP does not require rigorous surveillance protocols, such as those for urothelial carcinoma and exophytic urothelial papilloma. It is therefore important for the surgical pathologist to be aware of this rare entity in order to ensure correct patient management.

Keywords: Inverted urothelial papilloma, Upper urinary tract, Molecular markers, Microsatellite instability

\section{Background}

Inverted urothelial papilloma (IUP) is a rare lesion, histologically similar to inverted papilloma of the nasal cavity and paranasal sinuses. First reported in 1927 by Paschkis as "polypoid adenoma of the bladder" [1], it was later described in 1963 by Potts and Hirst as a distinct tumour entity of the urinary bladder [2]. IUP accounts for approximately $2 \%$ of all urothelial neoplasms.

\footnotetext{
* Correspondence: gabriella.nesi@unifi.it

${ }^{2}$ Pathology Section, Department of Health Sciences, University of Florence, Viale Pieraccini 6, 50139 Florence, Italy

Full list of author information is available at the end of the article
}

It usually occurs at the bladder neck, trigone or prostatic urethra, but is rare in the upper urinary tract. To the best of our knowledge, 68 IUP cases of the renal pelvis and ureter have been described in the English literature (Tables 1 and 2) [3-52].

Histological diagnosis of IUP can be difficult and several pathological conditions may enter differential diagnosis, including other urothelial neoplasms with endophytic growth patterns (i.e. papillary urothelial neoplasm of low malignant potential, low- and high-grade urothelial carcinoma), nested urothelial carcinoma, paraganglioma, carcinoid tumour, florid von Brunn nest 
Table 1 IUP of the renal pelvis (RP) previously reported in the English Literature (NS = Not Stated; NA = Not Assessed)

\begin{tabular}{|c|c|c|c|c|c|c|c|c|}
\hline Reference & Age & Sex & Presentation & Site & $\begin{array}{l}\text { Gross/ } \\
\text { Maximum } \\
\text { Diameter }(\mathrm{cm})\end{array}$ & $\begin{array}{l}\text { Associated Urothelial } \\
\text { Lesions }\end{array}$ & Treatment & $\begin{array}{l}\text { Recurrence (Follow- } \\
\text { Up) }\end{array}$ \\
\hline $\begin{array}{l}\text { Matz et al. } \\
\text { (1974) [3] }\end{array}$ & 68 & M & $\begin{array}{l}\text { Haematuria, } \\
\text { flank pain }\end{array}$ & Left RP & Nodule/1.5 & None & Nephroureterectomy & None (2 ys) \\
\hline $\begin{array}{l}\text { Assor (1976) } \\
{[4]}\end{array}$ & 79 & M & $\begin{array}{l}\text { Haematuria, } \\
\text { flank } \\
\text { discomfort }\end{array}$ & Right RP & $\begin{array}{l}\text { Sessile polyp/ } \\
1.5\end{array}$ & None & Partial resection & NS \\
\hline $\begin{array}{l}\text { Cameron } \\
\text { et al. (1976) } \\
\text { [5] }\end{array}$ & 58 & $\mathrm{~F}$ & NS & RP (side NS) & $\mathrm{NS} / 3$ & None & Nephroureterectomy & $\begin{array}{l}\text { Patient died of } \\
\text { carcinoma of the } \\
\text { endometrium four } \\
\text { years later }\end{array}$ \\
\hline $\begin{array}{l}\text { Di Cello et al. } \\
\text { (1980) [6] }\end{array}$ & 53 & M & Asymptomatic & $\begin{array}{l}\text { Left uretero-pelvic } \\
\text { junction }\end{array}$ & $\begin{array}{l}\text { Sessile polyp/ } \\
3\end{array}$ & None & Nephroureterectomy & NS \\
\hline $\begin{array}{l}\text { Theoret et al. } \\
\text { (1980) [7] }\end{array}$ & 89 & M & $\begin{array}{l}\text { Asymptomatic } \\
\text { (autopsy } \\
\text { finding) }\end{array}$ & RP (side NS) & NS & None & NA & NA \\
\hline $\begin{array}{l}\text { Uyama et al. } \\
\text { (1981) [8] }\end{array}$ & 73 & M & Haematuria & Left RP & $\mathrm{NS} / 2.5$ & None & $\begin{array}{l}\text { Nephroureterectomy, } \\
\text { radiation and } \\
\text { chemotherapy }\end{array}$ & None (5 ys) \\
\hline $\begin{array}{l}\text { Anderström } \\
\text { et al. (1982) } \\
{[9]}\end{array}$ & 62 & M & Asymptomatic & Left RP & Nodule/3 & $\begin{array}{l}\text { Synchronous grade } 2 \\
\text { transitional cell } \\
\text { carcinoma of } \\
\text { contralateral RP and } \\
\text { non-invasive grade } 2 \\
\text { transitional cell carcin- } \\
\text { oma of the bladder; } \\
\text { history of recurrent } \\
\text { grade } 2 \text { transitional } \\
\text { cell carcinoma of the } \\
\text { bladder }\end{array}$ & $\begin{array}{l}\text { Extracorporeal } \\
\text { resection of ureter } \\
\text { and RP and } \\
\text { autotransplant of } \\
\text { kidney to bladder }\end{array}$ & $\begin{array}{l}\text { Patient died of } \\
\text { metastatic poorly } \\
\text { differentiated } \\
\text { squamous cell } \\
\text { carcinoma of the } \\
\text { bladder three years } \\
\text { later; no recurrence } \\
\text { in the kidney where } \\
\text { IUP was diagnosed }\end{array}$ \\
\hline $\begin{array}{l}\text { Anderström } \\
\text { et al. (1982) } \\
\text { [9] }\end{array}$ & 49 & M & Ureteral colic & Right RP & Nodule/NS & None & NS & NS \\
\hline $\begin{array}{l}\text { Watters et al. } \\
\text { (1983) [10] }\end{array}$ & 65 & M & Haematuria & Left RP & $\begin{array}{l}\text { Pedunculated } \\
\text { polyp/1 }\end{array}$ & None & Nephroureterectomy & NS \\
\hline $\begin{array}{l}\text { Lausten et al. } \\
\text { (1984) [11] }\end{array}$ & 63 & M & NS & Right RP & $\begin{array}{l}\text { Pedunculated } \\
\text { polyp/1 }\end{array}$ & $\begin{array}{l}\text { Grade } 3 \text { invasive } \\
\text { polypoid transitional } \\
\text { cell carcinoma in the } \\
\text { contralateral RP after } 8 \\
\text { years }\end{array}$ & Nephrectomy & None (8.5 ys) \\
\hline $\begin{array}{l}\text { Taylor et al. } \\
\text { (1986) [12] }\end{array}$ & 65 & M & Haematuria & Right RP & $\begin{array}{l}\text { Sessile polyp/ } \\
\text { NS }\end{array}$ & None & Nephroureterectomy & None (2 ys) \\
\hline $\begin{array}{l}\text { Schulze et al. } \\
\text { (1986) [13] }\end{array}$ & 53 & M & Haematuria & Right RP & $\begin{array}{l}\text { Sessile polyp/ } \\
2.5\end{array}$ & None & Nephroureterectomy & NS \\
\hline $\begin{array}{l}\text { Schulze et al. } \\
\text { (1986) [13] }\end{array}$ & 55 & M & Haematuria & Left RP and ureter & $\begin{array}{l}\text { Not apparent } \\
\text { at gross } \\
\text { examination }\end{array}$ & None & Nephrectomy & NS \\
\hline $\begin{array}{l}\text { Romanelli } \\
\text { (1986) [14] }\end{array}$ & 52 & M & $\begin{array}{l}\text { Haematuria, } \\
\text { renal colic, }\end{array}$ & Right RP & $\begin{array}{l}\text { Sessile polyp/ } \\
2.1\end{array}$ & None & Nephroureterectomy & NS \\
\hline $\begin{array}{l}\text { Yamaguchi } \\
\text { et al. (1988) } \\
{[15]}\end{array}$ & 73 & M & Haematuria & Left RP & $\begin{array}{l}\text { Pedunculated } \\
\text { polyp/0.6 }\end{array}$ & $\begin{array}{l}\text { Synchronous low } \\
\text { grade transitional cell } \\
\text { carcinoma of the } \\
\text { bladder (ureteral } \\
\text { orifice) }\end{array}$ & Nephrectomy & None (1 y) \\
\hline $\begin{array}{l}\text { Schultz et al. } \\
\text { (1988) [16] }\end{array}$ & 58 & M & Haematuria & Left RP & NS & $\begin{array}{l}\text { Synchronous } \\
\text { superficial grade } 2 \\
\text { transitional cell } \\
\text { carcinoma of the } \\
\text { contralateral ureter } \\
\text { (nephroureterectomy }\end{array}$ & $\begin{array}{l}\text { Pyelotomy and } \\
\text { endoscopic resection }\end{array}$ & $\begin{array}{l}\text { IUP of the bladder } 1 \\
\text { y later }\end{array}$ \\
\hline
\end{tabular}


Table 1 IUP of the renal pelvis (RP) previously reported in the English Literature (NS = Not Stated; NA = Not Assessed) (Continued)

\begin{tabular}{|c|c|c|c|c|c|c|c|c|}
\hline Reference & Age & Sex & Presentation & Site & $\begin{array}{l}\text { Gross/ } \\
\text { Maximum } \\
\text { Diameter (cm) }\end{array}$ & $\begin{array}{l}\text { Associated Urothelial } \\
\text { Lesions }\end{array}$ & Treatment & $\begin{array}{l}\text { Recurrence (Follow- } \\
\text { Up) }\end{array}$ \\
\hline & & & & & & $\begin{array}{l}\text { with excision of the } \\
\text { bladder cuff) }\end{array}$ & & \\
\hline $\begin{array}{l}\text { Aubert et al. } \\
\text { (1988) [17] }\end{array}$ & 34 & M & Haematuria & Left RP & NS & None & Nephroureterectomy & None (18 months) \\
\hline $\begin{array}{l}\text { Kyriakos et al. } \\
\text { (1989) [18] }\end{array}$ & 73 & $\mathrm{~F}$ & Asymptomatic & $\begin{array}{l}\text { Multiple lesions: } \\
\text { Junction between } \\
\text { a upper pole } \\
\text { major calyx and } \\
\text { right RP (I); right } \\
\text { calix (II); distal } \\
\text { right ureter (III } \\
\text { and IV) }\end{array}$ & $\begin{array}{l}\text { Polyp/2.6 (I); } \\
\text { slightly } \\
\text { elevated } \\
\text { nodule/1 (II); } \\
\text { polyp/0.5 (III); } \\
\text { polyp/1.2 (IV) }\end{array}$ & None & Nephroureterectomy & None (11 months) \\
\hline $\begin{array}{l}\text { Bagley et al. } \\
\text { (1990) [19] }\end{array}$ & 64 & M & Haematuria & Right RP & Nodule/1 & $\begin{array}{l}\text { Recurrent transitional } \\
\text { cell carcinoma of the } \\
\text { bladder }\end{array}$ & $\begin{array}{l}\text { Ureteropyeloscopy } \\
\text { with endoscopic } \\
\text { resection }\end{array}$ & None (6 months) \\
\hline $\begin{array}{l}\text { Bassi et al. } \\
\text { (1991) [20] }\end{array}$ & 51 & M & $\begin{array}{l}\text { Haematuria, } \\
\text { flank pain }\end{array}$ & Left RP & $\begin{array}{l}\text { Sessile polyp/ } \\
0.5\end{array}$ & None & Partial resection & NS \\
\hline $\begin{array}{l}\text { Vlassopopulos } \\
\text { et al. (1992) } \\
\text { [21] }\end{array}$ & 59 & M & $\begin{array}{l}\text { Haematuria, } \\
\text { flank pain }\end{array}$ & Left RP & $\begin{array}{l}\text { Sessile polyp/ } \\
2\end{array}$ & None & Nephroureterectomy & None (12 months) \\
\hline $\begin{array}{l}\text { Ueda T et al. } \\
\text { (1992) [22] }\end{array}$ & 71 & M & Asymptomatic & Right RP & Nodule/4 & None & Nephrectomy & $\begin{array}{l}\text { Synchronous clear } \\
\text { cell carcinoma of } \\
\text { the homolateral } \\
\text { kidney, treated with } \\
\text { surgery and } \\
\text { anticancer drugs. } \\
\text { No recurrence from } \\
\text { IUP ( } 21 \text { months) }\end{array}$ \\
\hline $\begin{array}{l}\text { Spevack et al. } \\
\text { (1995) [23] }\end{array}$ & 64 & M & Haematuria & Right RP & $\begin{array}{l}\text { Pedunculated } \\
\text { polyp } / 2.5\end{array}$ & None & Partial resection & None (42 months) \\
\hline $\begin{array}{l}\text { Chiura et al. } \\
\text { (1998) [24] }\end{array}$ & 63 & M & Haematuria & Right RP & $N S / 3$ & $\begin{array}{l}\text { Transitional cell } \\
\text { carcinoma of the left } \\
\text { distal ureter three } \\
\text { years later, treated } \\
\text { with surgery, radiation } \\
\text { therapy and } \\
\text { chemotherapy }\end{array}$ & Nephroureterectomy & $\begin{array}{l}\text { None ( } 1 \text { y after } \\
\text { surgery for } \\
\text { carcinoma) }\end{array}$ \\
\hline $\begin{array}{l}\text { Chiura et al. } \\
\text { (1998) [24] }\end{array}$ & 53 & M & Haematuria & $\mathrm{RP}$ & NS & Pyelitis cystica & Nephroureterectomy & NS \\
\hline $\begin{array}{l}\text { Chiura et al. } \\
\text { (1998) [24] }\end{array}$ & 64 & M & Asymptomatic & Right RP & NS & $\begin{array}{l}\text { Recurrent transitional } \\
\text { cell carcinoma of the } \\
\text { bladder (previous and } \\
\text { subsequent to IUP } \\
\text { diagnosis) }\end{array}$ & $\begin{array}{l}\text { Ureteroscopy and } \\
\text { biopsy }\end{array}$ & $\begin{array}{l}\text { Transitional cell } \\
\text { carcinoma in the } \\
\text { homolateral kidney } \\
\text { and ureter } 9 \text { ys later }\end{array}$ \\
\hline $\begin{array}{l}\text { Darras et al. } \\
\text { (2005) [25] }\end{array}$ & 52 & M & $\begin{array}{l}\text { Haematuria, } \\
\text { occasional } \\
\text { discomfort in } \\
\text { the lower } \\
\text { abdomen }\end{array}$ & Left RP & Polyp/NS & $\begin{array}{l}\text { Synchronous IUP of } \\
\text { the bladder }\end{array}$ & Partial resection & None (NS) \\
\hline $\begin{array}{l}\text { Luo et al. } \\
\text { (2012) [26] }\end{array}$ & 62 & M & Asymptomatic & Right RP & $\begin{array}{l}\text { Pedunculated } \\
\text { polyp }\end{array}$ & None & Nephroureterectomy & None (NS) \\
\hline $\begin{array}{l}\text { Luo et al. } \\
\text { (2012) [26] }\end{array}$ & 66 & M & Haematuria & Left RP & $\begin{array}{l}\text { Pedunculated } \\
\text { polyp }\end{array}$ & None & Nephroureterectomy & None (NS) \\
\hline $\begin{array}{l}\text { Luo et al. } \\
\text { (2012) [26] }\end{array}$ & 64 & M & Haematuria & Left RP & $\begin{array}{l}\text { Pedunculated } \\
\text { polyp }\end{array}$ & None & Nephroureterectomy & None (NS) \\
\hline $\begin{array}{l}\text { Luo et al. } \\
\text { (2012) [26] }\end{array}$ & 73 & $\mathrm{~F}$ & Flank pain & Right RP & $\begin{array}{l}\text { Pedunculated } \\
\text { polyp }\end{array}$ & None & Nephroureterectomy & None (NS) \\
\hline
\end{tabular}


Table 2 IUP of the ureter $(U)$ previously reported in the English Literature (NS = Not Stated; NA = Not Assessed)

\begin{tabular}{|c|c|c|c|c|c|c|c|c|}
\hline Reference & Age & Sex & Presentation & Site & $\begin{array}{l}\text { Gross/Maximum } \\
\text { Diameter }(\mathrm{cm})\end{array}$ & Associated Urothelial Lesions & Treatment & $\begin{array}{l}\text { Rrecurrence } \\
\text { (Follow-Up) }\end{array}$ \\
\hline $\begin{array}{l}\text { Geisler et al. } \\
\text { (1980) [27] }\end{array}$ & 77 & M & Flank pain & $\begin{array}{l}\text { Left } \\
\text { middle U }\end{array}$ & Pedunculated/2.5 & None & NephroUectomy & NS \\
\hline $\begin{array}{l}\text { Silverstein et al. } \\
\text { (1981) [28] }\end{array}$ & 65 & M & Asymptomatic & $\begin{array}{l}\text { Left } \\
\text { middle } U\end{array}$ & Pedunculated/2.5 & None & Partial resection & NS \\
\hline $\begin{array}{l}\text { Silverstein et al. } \\
\text { (1981) [28] }\end{array}$ & 68 & M & Haematuria & $\begin{array}{l}\text { Right } \\
\text { middle U }\end{array}$ & Polypoid/2.5 & None & NephroUectomy & NS \\
\hline $\begin{array}{l}\text { Fromowitz } \\
\text { et al. (1981) } \\
\text { [29] }\end{array}$ & 75 & M & Haematuria & $\begin{array}{l}\text { Right } U \text {, at } \\
\text { junction of } \\
\text { proximal } \\
\text { and } \\
\text { middle } \\
\text { thirds }\end{array}$ & Flat, polypoid/1.8 & None & NephroUectomy & NS \\
\hline $\begin{array}{l}\text { Fromowitz } \\
\text { et al. (1981) } \\
\text { [29] }\end{array}$ & 56 & M & Asymptomatic & $\begin{array}{l}\text { Right } \\
\text { distal U }\end{array}$ & Raised/1.1 & $\begin{array}{l}\text { Adenocarcinoma of the } \\
\text { bladder } 7 \text { months later with } \\
\text { three recurrences during next } 2 \\
\text { ys }\end{array}$ & NephroUectomy & None (2 ys) \\
\hline $\begin{array}{l}\text { Ajrawat et al. } \\
\text { (1982) [30] }\end{array}$ & 86 & $\mathrm{~F}$ & Flank pain & $\begin{array}{l}\text { Right } \\
\text { distal U }\end{array}$ & $\begin{array}{l}\text { Lobulated mass/ } \\
1.5\end{array}$ & None & Partial resection & NS \\
\hline $\begin{array}{l}\text { Naito et al. } \\
\text { (1983) [31] }\end{array}$ & 68 & M & Haematuria & $\begin{array}{l}\text { Right } \\
\text { distal U }\end{array}$ & Pedunculated/1.5 & None & NephroUectomy & None (2 ys) \\
\hline $\begin{array}{l}\text { Jacobellis et al. } \\
\text { (1983) [32] }\end{array}$ & 59 & $\mathrm{~F}$ & $\begin{array}{l}\text { Haematuria, } \\
\text { flank pain }\end{array}$ & $\begin{array}{l}\text { Left } \\
\text { lumbar U }\end{array}$ & Sessile/3 & $\begin{array}{l}\text { Synchronous conventional } \\
\text { papilloma of homolateral lower } \\
\text { calix }\end{array}$ & NephroUectomy & NS \\
\hline $\begin{array}{l}\text { Embon et al. } \\
\text { (1984) [33] }\end{array}$ & 69 & M & Haematuria & $\begin{array}{l}\text { Right } \\
\text { distal U }\end{array}$ & Polypoid/3 & None & Partial resection & $\begin{array}{l}\text { None (9 } \\
\text { months) }\end{array}$ \\
\hline $\begin{array}{l}\text { Lausten et al. } \\
\text { (1984) [11] }\end{array}$ & 60 & M & Asymptomatic & $\begin{array}{l}\text { Right } \\
\text { proximal } \\
\cup\end{array}$ & $\begin{array}{l}\text { Sessile tumour/ } \\
0.3\end{array}$ & $\begin{array}{l}\text { Grade } 2 \text { non-invasive transi- } \\
\text { tional cell papilloma located } \\
\text { above the homolateral Uic ori- } \\
\text { fice } 1 \text { and half years earlier }\end{array}$ & $\begin{array}{l}\text { Cranial } \\
\text { heminephroUectomy }\end{array}$ & $\begin{array}{l}\text { None (19 } \\
\text { months) }\end{array}$ \\
\hline $\begin{array}{l}\text { Lausten et al. } \\
\text { (1984) [11] }\end{array}$ & 71 & M & $\begin{array}{l}\text { Flank pain } \\
\text { (prostatism) }\end{array}$ & $\begin{array}{l}\text { Right } \\
\text { proximal } \\
\cup\end{array}$ & $\begin{array}{l}\text { Pedunculated } \\
\text { tumour/ } 1\end{array}$ & None & Partial U resection & $\begin{array}{l}\text { None (18 } \\
\text { months) }\end{array}$ \\
\hline $\begin{array}{l}\text { Perrin et al. } \\
\text { (1984) [34] }\end{array}$ & 63 & M & $\begin{array}{l}\text { Haematuria, } \\
\text { renal colic }\end{array}$ & $\begin{array}{l}\text { Left } \\
\text { middle U }\end{array}$ & Polypoid/NS & None & Partial resection & $\begin{array}{l}\text { Dead after } 2 \text { ys } \\
\text { of cirrhosis; no } \\
\text { recurrence of } \\
\text { Ual lesion }\end{array}$ \\
\hline $\begin{array}{l}\text { Mottola et al. } \\
\text { (1984) [35] }\end{array}$ & 56 & M & $\begin{array}{l}\text { Haematuria, } \\
\text { flank pain }\end{array}$ & $\begin{array}{l}\text { Right } \\
\text { lumbar U }\end{array}$ & NS & None & Partial resection & $\begin{array}{l}\text { None (12 } \\
\text { months) }\end{array}$ \\
\hline $\begin{array}{l}\text { Palvio (1985) } \\
{[36]}\end{array}$ & 50 & M & Haematuria & $\begin{array}{l}\text { Distal } \\
\text { portion of } \\
\text { the left U } \\
\text { (above the } \\
\text { Ual orifice) }\end{array}$ & $\begin{array}{l}\text { Pedunculated } \\
\text { tumour/ NS }\end{array}$ & $\begin{array}{l}\text { After } 8 \text { ys from the first } \\
\text { diagnosis of IUP of the distal } U \text {, } \\
\text { the patient underwent } \\
\text { nephroUectomy for two lesions } \\
\text { at the Uopelvic junction and in } \\
\text { the distal part of the } U \text { (IUP } \\
\text { with areas of non-invasive tran- } \\
\text { sitional cell carcinoma, grade 2) }\end{array}$ & TUR & Yes, after 3 ys \\
\hline $\begin{array}{l}\text { Moss et al. } \\
\text { (1987) [37] }\end{array}$ & 79 & M & Asymptomatic & Right U & $\mathrm{NS} / 1$ & None & $\begin{array}{l}U \text { resection during } \\
\text { hemicolectomy }\end{array}$ & $\begin{array}{l}\text { None (3 } \\
\text { months) }\end{array}$ \\
\hline $\begin{array}{l}\text { Corkill et al. } \\
\text { (1987) [38] }\end{array}$ & 62 & M & Haematuria & $\begin{array}{l}\text { Left distal } \\
\cup\end{array}$ & Polypoid/0.8 & None & Partial resection & None (7ys) \\
\hline $\begin{array}{l}\text { Duchek et al. } \\
\text { (1987) [39] }\end{array}$ & 24 & M & $\begin{array}{l}\text { Haematuria, } \\
\text { renal colic }\end{array}$ & $\begin{array}{l}\text { Right } \\
\text { middle U }\end{array}$ & $\begin{array}{l}\text { Pedunculated } \\
\text { lesion/NS }\end{array}$ & None & Local resection & None (5 ys) \\
\hline $\begin{array}{l}\text { Abulafi A et al. } \\
\text { (1987) [40] }\end{array}$ & 62 & M & Haematuria & $\begin{array}{l}\text { Right } \\
\text { proximal } \\
\cup\end{array}$ & $\begin{array}{l}\text { Pedunculated } \\
\text { lesion/NS }\end{array}$ & None & Local resection & NS \\
\hline $\begin{array}{l}\text { Villani U et al. } \\
\text { (1987) [41] }\end{array}$ & 56 & M & Haematuria & $\begin{array}{l}\text { Left pelvic } \\
U\end{array}$ & NS & $\begin{array}{l}\text { Synchronous grade } 2 \text { papillary } \\
\text { transitional cell carcinoma of }\end{array}$ & Local resection & None (1y) \\
\hline
\end{tabular}


Table 2 IUP of the ureter $(U)$ previously reported in the English Literature (NS = Not Stated; NA = Not Assessed) (Continued)

\begin{tabular}{|c|c|c|c|c|c|c|c|c|}
\hline Reference & Age & Sex & Presentation & Site & $\begin{array}{l}\text { Gross/Maximum } \\
\text { Diameter }(\mathrm{cm})\end{array}$ & Associated Urothelial Lesions & Treatment & $\begin{array}{l}\text { Rrecurrence } \\
\text { (Follow-Up) }\end{array}$ \\
\hline & & & & & & the bladder & & \\
\hline $\begin{array}{l}\text { Kostakopolulos } \\
\text { et al. (1988) } \\
\text { [42] }\end{array}$ & 66 & M & $\begin{array}{l}\text { Haematuria, } \\
\text { renal colic }\end{array}$ & Left U & NS & None & Partial resection & $\begin{array}{l}\text { None (6 } \\
\text { months) }\end{array}$ \\
\hline $\begin{array}{l}\text { Garritano et al. } \\
\text { (1988) [43] }\end{array}$ & 49 & M & Haematuria & $\begin{array}{l}\text { Left } \\
\text { middle U }\end{array}$ & $\begin{array}{l}\text { Pedunculated } \\
\text { lobulated } \\
\text { tumour/3 }\end{array}$ & None & Local resection & None (5 ys) \\
\hline $\begin{array}{l}\text { Aubert et al. } \\
\text { (1988) [17] }\end{array}$ & 71 & M & $\begin{array}{l}\text { Haematuria, } \\
\text { flank pain }\end{array}$ & $\begin{array}{l}\text { Right } \\
\text { lower U }\end{array}$ & NS & None & Partial resection & None (5 ys) \\
\hline $\begin{array}{l}\text { Page et al. } \\
\text { (1991) [44] }\end{array}$ & 56 & M & Haematuria & $\begin{array}{l}\text { Distal } U, \\
\text { bilateral }\end{array}$ & $\begin{array}{l}\text { Multiple sessile } \\
\text { lesions/right side } \\
\text { lesion: } 3 \mathrm{~cm} ; 2 \\
\text { lesions of the left } \\
\text { side: } 2 \mathrm{~cm} \text { each) }\end{array}$ & None & $\begin{array}{l}\text { Right side: partial } \\
\text { Uectomy; Left side: } \\
\text { complete Uectomy }\end{array}$ & NS \\
\hline $\begin{array}{l}\text { Kunimi et al. } \\
\text { (1994) [45] }\end{array}$ & 42 & M & Flank pain & $\begin{array}{l}\text { Left } \\
\text { middle U }\end{array}$ & $\begin{array}{l}\text { Pedunculated } \\
\text { polyp/ } 2.7\end{array}$ & $\begin{array}{l}\text { Superficial transitional cell } \\
\text { carcinoma grade } 2 \text { of the } \\
\text { bladder ( } 23 \text { months later) }\end{array}$ & NephroUectomy & $\begin{array}{l}\text { None }(20 \\
\text { months after } \\
\text { the diagnosis of } \\
\text { carcinoma) }\end{array}$ \\
\hline $\begin{array}{l}\text { de Knijff et al. } \\
\text { (1997) [46] }\end{array}$ & 63 & M & $\begin{array}{l}\text { Urinary } \\
\text { frequency } \\
\text { and urge }\end{array}$ & $\begin{array}{l}\text { Right } \\
\text { distal U }\end{array}$ & $\mathrm{NS} / 2$ & $\begin{array}{l}\text { Invasive bladder tumour six } \\
\text { years later, treated with } \\
\text { cystoprostatectomy }\end{array}$ & Local resection & None \\
\hline $\begin{array}{l}\text { Hoekx et al. } \\
\text { (1998) [47] }\end{array}$ & 71 & M & $\begin{array}{l}\text { Haematuria, } \\
\text { flank pain }\end{array}$ & $\begin{array}{l}\text { Left distal } \\
\cup \text { and } \\
\text { right distal } \\
\cup\end{array}$ & $\begin{array}{l}\text { Smooth surface/ } \\
\text { NS }\end{array}$ & $\begin{array}{l}\text { Synchronous grade } 2 \\
\text { transitional cell carcinoma of } \\
\text { the bladder (T1NOMO) }\end{array}$ & $\begin{array}{l}\text { Left partial resection } \\
\text { and right } \\
\text { nephroUectomy }\end{array}$ & $\begin{array}{l}\text { Multiple } \\
\text { recurrences of } \\
\text { urinary badder } \\
\text { carcinoma } \\
\text { (duration of } \\
\text { follow-up NS) }\end{array}$ \\
\hline $\begin{array}{l}\text { Lyon et al. } \\
\text { (2006) [48] }\end{array}$ & 59 & M & Haematuria & $\begin{array}{l}\text { Left } \\
\text { proximal } \\
\cup\end{array}$ & Sessile lesion/2.5 & None & Local resection & None (1 y) \\
\hline $\begin{array}{l}\text { Kilciler et al. } \\
\text { (2008) [49] }\end{array}$ & 62 & M & $\begin{array}{l}\text { Haematuria, } \\
\text { flank pain }\end{array}$ & $\begin{array}{l}\text { Middle U } \\
\text { (side NS) }\end{array}$ & $\mathrm{NS} / 2$ & None & NephroUectomy & None (NS) \\
\hline $\begin{array}{l}\text { Mertziotis et al. } \\
\text { (2012) [50] }\end{array}$ & 62 & M & $\begin{array}{l}\text { Haematuria, } \\
\text { flank pain }\end{array}$ & $\begin{array}{l}\text { Right } \\
\text { upper U }\end{array}$ & $\begin{array}{l}\text { Exophytic lesion/ } \\
4\end{array}$ & None & Nephrouretectomy & $\begin{array}{l}\text { None (14 } \\
\text { months) }\end{array}$ \\
\hline $\begin{array}{l}\text { Murtaza et al. } \\
\text { (2012) [51] }\end{array}$ & 35 & M & Flank pain & $\begin{array}{l}\text { Left distal } \\
\cup\end{array}$ & $\begin{array}{l}\text { Multiple small to } \\
\text { large polypoid } \\
\text { lesions }\end{array}$ & None & Local resection & $\begin{array}{l}\text { None (6 } \\
\text { months) }\end{array}$ \\
\hline $\begin{array}{l}\text { Lopez-Fontana } \\
\text { et al. (2012) } \\
\text { [52] }\end{array}$ & 30 & M & Haematuria & $\begin{array}{l}\text { Right } \\
\text { distal U }\end{array}$ & $\begin{array}{l}\text { Polypoid lesion/ } \\
1.6\end{array}$ & None & Partial Uectomy & $\begin{array}{l}\text { None (4 } \\
\text { months) }\end{array}$ \\
\hline $\begin{array}{l}\text { Luo et al. } \\
\text { (2012) [26] }\end{array}$ & 70 & M & Haematuria & Right U & Pedunculated & None & NephroUectomy & None (NS) \\
\hline $\begin{array}{l}\text { Luo et al. } \\
\text { (2012) [26] }\end{array}$ & 61 & M & Flank Pain & Left U & Pedunculated & Not specified & Partial Uctomy & None (NS) \\
\hline $\begin{array}{l}\text { Luo et al. } \\
\text { (2012) [26] }\end{array}$ & 67 & M & Asymptomatic & Left U & $\begin{array}{l}\text { Multiple lesions/ } \\
\text { Pedunculated }\end{array}$ & None & NephroUectomy & None (NS) \\
\hline $\begin{array}{l}\text { Luo et al. } \\
\text { (2012) [26] }\end{array}$ & 67 & M & Haematuria & Left U & $\begin{array}{l}\text { Multiple lesions/ } \\
\text { Pedunculated }\end{array}$ & None & Local resection & None (NS) \\
\hline $\begin{array}{l}\text { Luo et al. } \\
\text { (2012) [26] }\end{array}$ & 73 & M & Haematuria & Left U & Pedunculated & Not specified & Partial Uctomy & None (NS) \\
\hline $\begin{array}{l}\text { Luo et al. } \\
\text { (2012) [26] }\end{array}$ & 68 & M & Haematuria & Left U & Pedunculated & Not specified & Partial Uctomy & None (NS) \\
\hline
\end{tabular}


proliferation and cystitis cystica et glandularis. Most of the investigated immunohistochemical markers are of little use in routine practice, and microscopic assessment remains the current gold standard. IUPs are benign tumours and can be successfully treated by conservative surgery. While specific molecular alterations are well described for papillary urothelial neoplasms, only few studies have been conducted on inverted lesions, suggesting a correlation between inverted growth and mismatch repair deficiency in urothelial carcinoma of the upper urinary tract [53].

Two additional cases of polypoid IUP of the renal pelvis and the ureter are herein presented with a systematic review of the literature.

\section{Clinical cases \\ Case 1}

A 76-year-old woman was admitted with persistent right flank pain and macroscopic haematuria. A computed tomography $(\mathrm{CT})$ scan revealed a 2 -cm polypoid lesion in the right renal pelvis, causing mild proximal hydronephrosis. The patient was otherwise in good health and advised to undergo nephroureterectomy due to the high likelihood of malignancy. Postoperative course was unremarkable, and the patient was discharged eight days after admission. She is alive and free of disease six years after treatment.

\section{Case 2}

A 56-year-old man presented with gross haematuria. A CT scan demonstrated a filling defect in the lower third of the right ureter with no evidence of lithiasis. Owing to the distal location of the lesion, segmentary ureterectomy was performed. The patient is asymptomatic five years after complete excision of the tumour.

\section{Pathological findings}

Both cases displayed similar gross and histological features. In case 1 , a sessile polypoid tumour measured 2 $\mathrm{cm}$ in greatest diameter. Case 2 presented as a $1.4-\mathrm{cm}$ polypoid mass with a thin stalk. Microscopically, both lesions consisted of anastomosing trabeculae and cords growing downward into the lamina propria and lacked any true exophytic papillary component. Prominent peripheral palisading was seen in the trabeculae. There was no evidence of significant nuclear atypia and less than $1 /$ 10 high-power field mitotic figures were found. Hyalinised collagenous stroma was seen in case 1 . Microcyst formation and foci of squamous metaplasia were occasionally observed in case 2. Histology was consistent with IUP (Fig. 1).

Representative sections of the lesions were selected for immunohistochemical analysis. As primary antibodies, we used rabbit monoclonal Ki-67 (clone 30.9, ready to use; Ventana, Tucson, AZ), rabbit monoclonal CK20
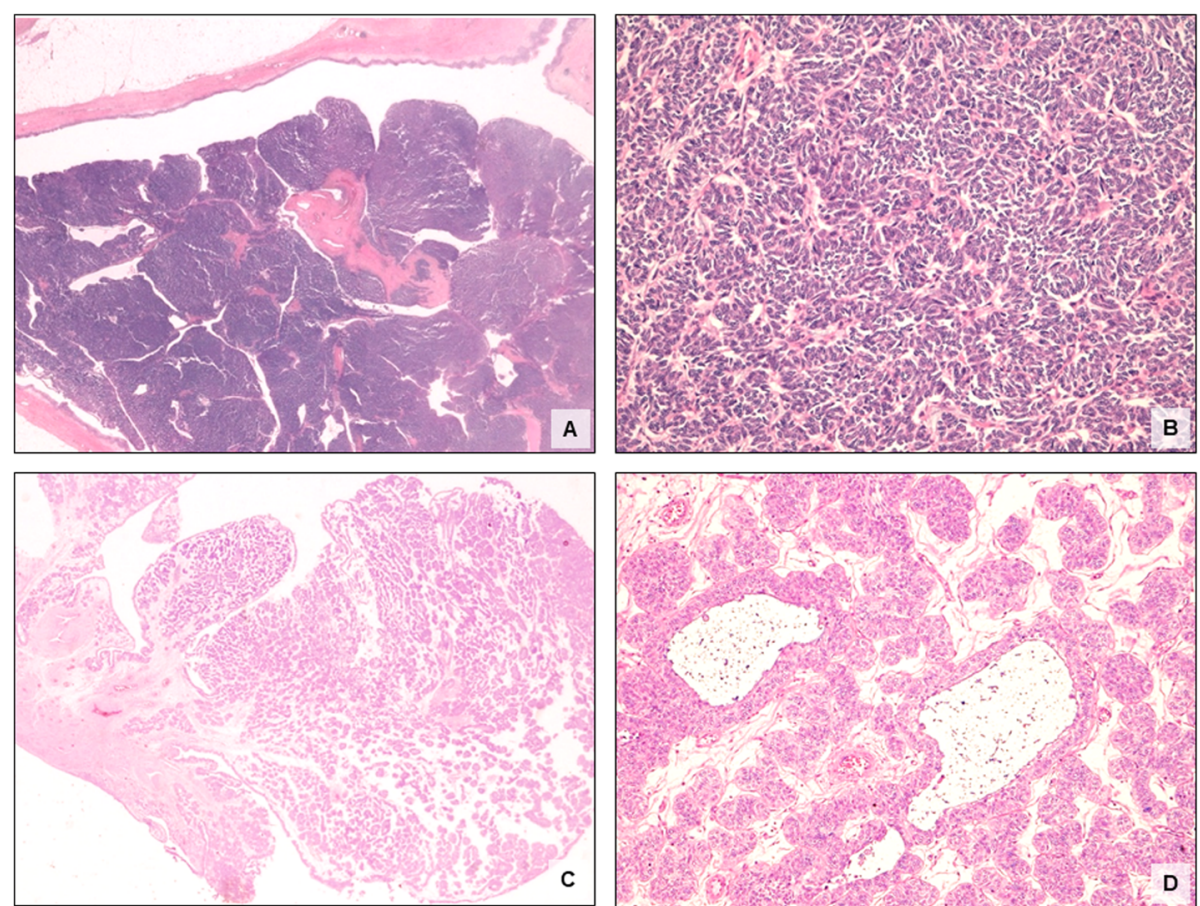

Fig. 1 Histological features of two cases of IUP of the upper urinary tract. Sessile polypoid tumour of the renal pelvis consisting of anastomosing trabeculae and cords growing downward into the lamina propria, with prominent peripheral palisading in the trabeculae (Case 1: a, b). Pedunculated polypoid IUP of the distal ureter characterized by microcyst formation and foci of squamous metaplasia (Case 2: $\mathbf{c}$, d) 
(clone SP33, ready to use; Ventana), mouse monoclonal PMS2 (clone A16-4, ready to use; Ventana), mouse monoclonal MLH1 (clone M1, ready to use; Ventana), mouse monoclonal MSH2 (clone G219-1129, ready to use; Ventana) and rabbit monoclonal MSH6 (clone SP93, ready to use; Ventana). Sections were stained on a Ventana BenchMark ULTRA immunostainer (Ventana Medical Systems). The procedure involved pretreatment with Cell Conditioning 1 followed by antibody incubation. The signal was then developed with ultraView Universal DAB Detection Kit for antibodies against Ki-67 and CK20. OptiView DAB IHC Detection Kit was employed for all other antibodies.

Both lesions were negative for CK20 and exhibited uniformly low Ki-67 (<1\%) (Fig. 2). Expression of the mismatch-repair protein was considered positive if at least $10 \%$ of neoplastic cells showed nuclear staining [54]. Loss of MSH6 was seen in both cases, alongside with retention of MLH1, MSH2, and PMS2 expression (Fig. 3).

\section{Discussion}

IUP of the upper urinary tract is a benign tumour with 68 cases described to date in the English literature. It usually manifests in middle-aged adults within the 6th or 7th decade of life, and males are more commonly affected than females [26].

The most frequent presenting symptoms are haematuria, macroscopic or microscopic, and renal colic.
Irritative symptoms, as well as urinary tract obstruction, have also been reported [55]. In a high percentage of cases, however, tumours are asymptomatic and detected during unrelated clinical investigations.

Preoperative diagnosis of IUP is difficult. Imaging studies may reveal non-specific findings such as filling defects of obstructive masses, often associated with hydronephrosis, hydroureter or renal stones [56]. Cytological morphology falls within the range of normal or mild atypia since IUP is covered by a normal and intact mucosal layer. Accurate preoperative diagnosis requires biopsy and visualisation through endoscopic examination. These procedures also provide therapeutic indications, thus avoiding unnecessary nephroureterectomy [26]. Due to the high likelihood of malignancy, preoperative biopsies were not carried out in our cases and patients underwent radical surgery.

Grossly, IUP presents as a solid or polypoid mass with smooth mucosal, non-papillary covering surface. Most tumours measure less than $3 \mathrm{~cm}$ in diameter but can reach up to $8 \mathrm{~cm}$ or more. They usually occur as solitary lesions, although $3.6-6 \%$ are bilateral or multicentric [55].

Histologically, IUP is characterised by endophytic growth of epithelial elements arranged in nests and cords, growing down from the surface urothelium into the lamina propria with expansible borders. Cystic areas and foci of squamous metaplasia are common. Neither fibrovascular cores nor desmoplasia are seen in IUP and
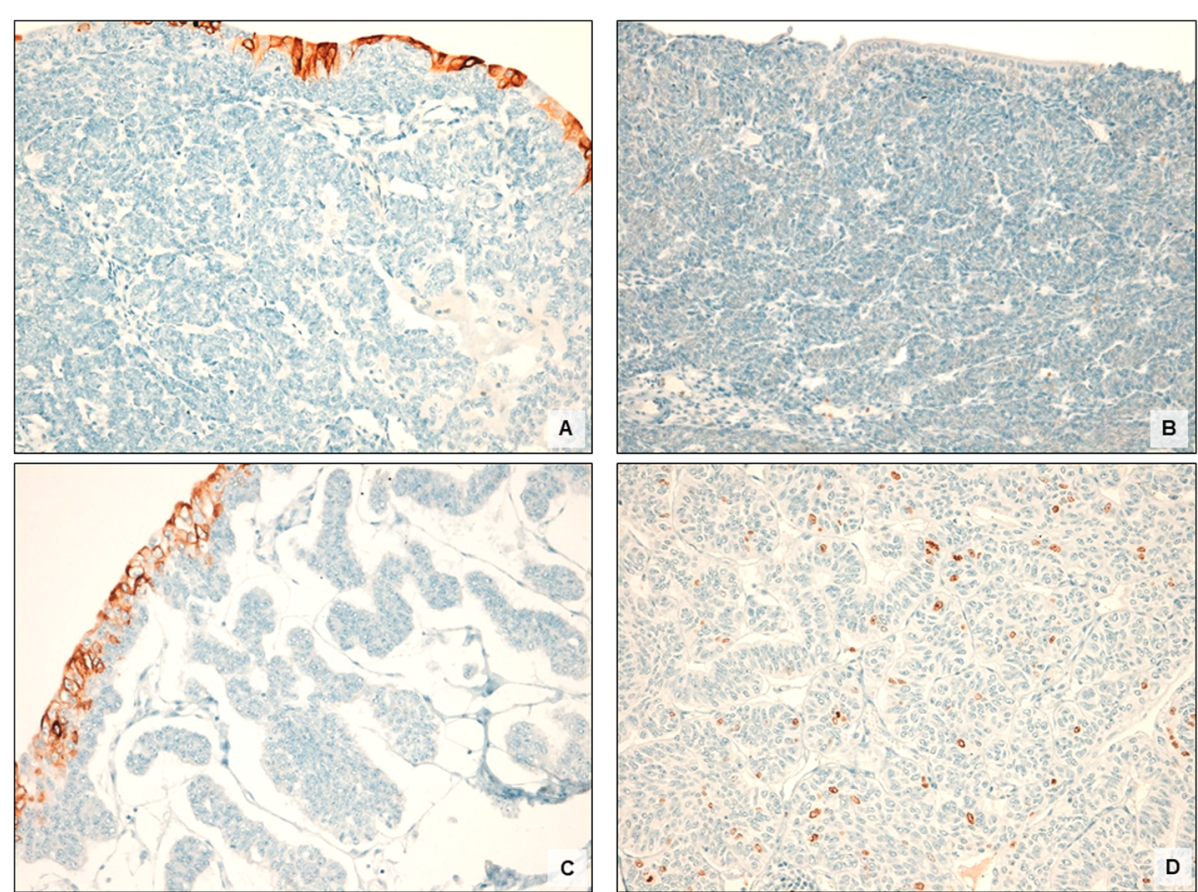

Fig. 2 Immunohistochemical results in two cases of IUP pf the upper urinary tract. Both cases were negative for CK20 immunostaining (Case 1: a; Case 2: c) and showed low Ki-67 labelling index (<1\%) (Case 2: b; Case 2: d) 


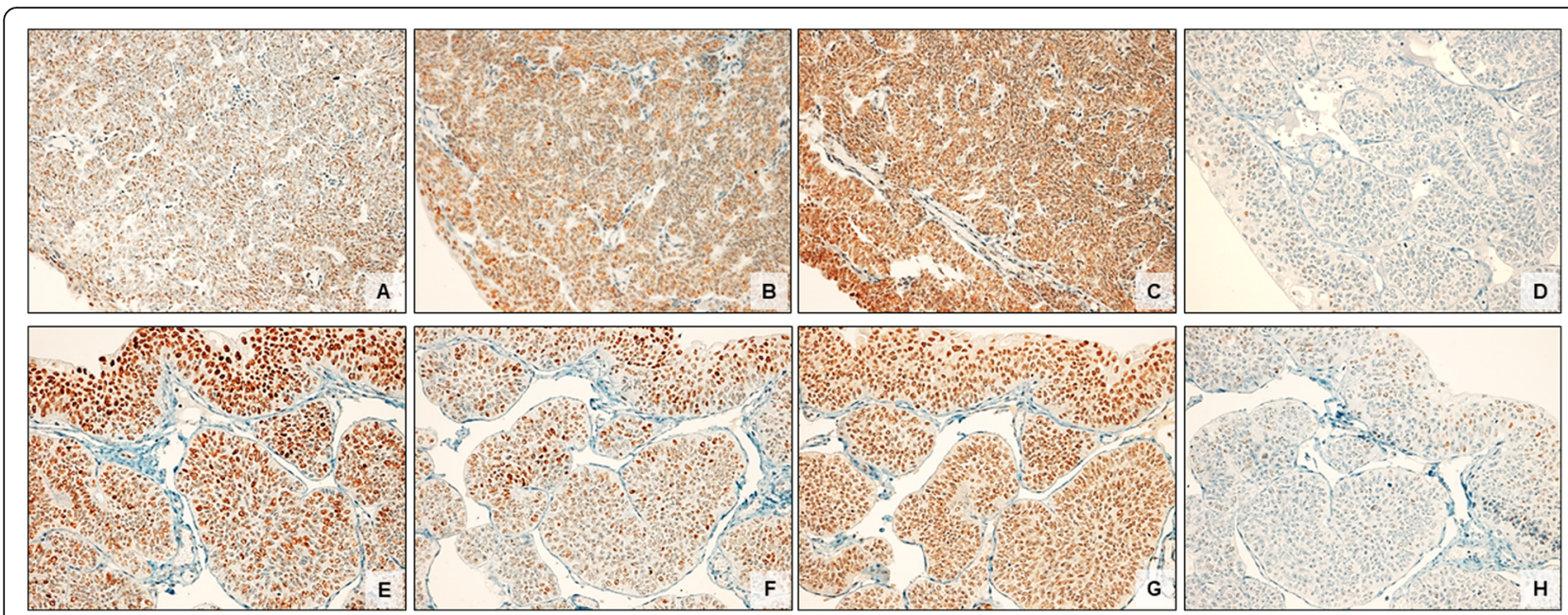

Fig. 3 Expression of the mismatch-repair proteins in two cases of IUP of the upper urinary tract (Case 1: a-d; Case 2: e-h). Nuclear staining for MLH1 $(\mathbf{a}, \mathbf{e})$, MSH2 (b, f), PMS2 (c, g) was observed in both cases, whereas the tumours showed loss of MSH6 expression (d, $\mathbf{h})$

stromal inflammation is minimal. Necrosis and mitotic activity are absent. Distinction between inverted papilloma and urothelial carcinoma with an endophytic growth pattern can be challenging. Contrary to IUP, urothelial carcinoma with inverted configuration shows cytological atypia, mitoses, nuclear pleomorphism and often displays an exophytic papillary component. In addition, invasion into the muscularis propria may occur in urothelial carcinoma but not in IUP. When biopsies are of small size or morphological artefacts and tangential sectioning obscure the lesion, differentiating between these biologically different entities becomes increasingly difficult [57].

Recently, Wobker et al. described 13 cases of a unique urothelial tumour occurring exclusively in the renal pelvis and ureter, named polypoid urothelial proliferation with inverted growth pattern (PUTIP). Morphologically, PUTIP exhibits hybrid features between a totally inverted PUNLMP, IUP and florid proliferation of von Brunn nests [58]. PUTIP may show a distinct inverted papilloma-like component with densely hyalinised collagenous stroma, but lacks the thin anastomosing cords typical of IUP.

In the present study, we observed low Ki-67 proliferation index and negativity for CK20 in both cases. A number of immunohistochemical markers have been shown to be frequently expressed in urothelial carcinomas, including the proliferation marker $\mathrm{Ki}-67$ and CK20 [59]. IUP may be aneuploid and demonstrate high proliferative activity, although these features do not necessarily correlate with malignant behaviour $[60,61]$.

Our cases showed loss of MSH6 by immunohistochemistry, whereas expression of MSH2, MLH1 and PMS2 was retained. The molecular genetic abnormalities of IUP appear to differ from those of urothelial carcinoma, suggesting that these two neoplasms are unrelated [62]. Inverted-type urothelial carcinomas of the renal pelvis can be associated with MSI. Hartmann and co-authors examined 132 urothelial carcinomas of the upper urinary tract exhibiting some degree of inverted growth, and found that 35 (26.5\%) were microsatellite unstable by polymerase chain reaction analysis [53]. Similar results were obtained by Harper in 214 patients with upper tract urothelial carcinoma tested for mismatch repair protein loss by immunohistochemistry [63]. In a multicentric study conducted on 62 IUPs of the urinary bladder Eiber and co-authors demonstrated aberrant immunostaining for MSH2 (5.8\%), MLH1 (11.8\%) and MSH6 (3.8\%) [62]. As previously described, cellular loss of one MMR protein is not sufficient to cause detectable microsatellite defects [64]. Therefore, our observation may be spurious and unrelated to microsatellite instability, and should be confirmed in a larger series of IUPs of the upper urinary tract. In addition, our patients did not show any stigmata of Lynch syndrome or HNPCC-associated background.

Regarding treatment options, nephroureterectomy, local resection or partial ureterectomy with preservation of the kidney, and endoscopic surgery may be of use [65]. After excision, some authors recommend a followup protocol (endoscopy and radiographical studies) similar to that used in patients with low-grade urothelial carcinoma [26], while others do not advocate this rigorous and long-term follow-up due to the low risk of recurrence and favourable prognosis of IUP [66].

In conclusion, IUP of the upper urinary tract is an extremely rare tumour characterised by an inverted pattern of growth and constituted by normal to minimally atypical proliferating urothelium. The absence of progression of IUP on long-term follow-up argues against the need 
of patients' continuous surveillance when strict diagnostic criteria are followed, a complete resection can be ascertained and no history of previous or concurrent urothelial malignancies is recorded.

\section{Abbreviations}

IUP: Inverted Urothelial Papilloma; CT: Computed Tomography; PUTIP: Polypoid Urothelial Proliferation with Inverted Growth Pattern; RP: Renal Pelvis; NS: Not Stated; NA: Not Applicable

\section{Acknowledgements}

None.

\section{Authors' contributions}

RS conceived and designed the study, evaluated histological slides and contributed in writing the manuscript. ICG contributed in evaluating histological slides, collecting the data and writing the manuscript. JIL contributed with a case and participated to the design and implementation of the research. GN contributed to the design and implementation of the research, to the analysis of the results and in writing the manuscript. VC contributed in writing the manuscript. The author(s) read and approved the final manuscript.

\section{Funding}

The authors received no financial support for the research, authorship, and/ or publication of this article.

\section{Availability of data and materials}

The authors confirm that the data supporting the findings of this study are available within the article.

\section{Compliance with ethical standards}

All procedures followed were in accordance with the ethical standards of the responsible committee on human experimentation (institutional and national) and with the Helsinki Declaration of 1975, as revised in 2000.

\section{Consent for publication}

All authors have agreed with the submission in its present form.

\section{Competing interests}

No conflict of interest has been declared.

\section{Author details}

${ }^{1}$ Pathology Unit, Careggi University Hospital, Florence, Italy. ${ }^{2}$ Pathology Section, Department of Health Sciences, University of Florence, Viale Pieraccini 6, 50139 Florence, Italy. ${ }^{3}$ Centro di Riferimento Oncologico di Aviano (CRO), IRCCS, Aviano, Italy. ${ }^{4}$ Department of Medical, Surgical and Health Sciences, University of Trieste Medical School, Trieste, Italy. ${ }^{5}$ University of the Basque Country, Barakaldo, Spain.

Received: 7 January 2020 Accepted: 8 April 2020

Published online: 22 April 2020

\section{References}

1. Paschkis R. Uber Adenoma der Harnblase. Z Urol Chir. 1927;21:315-25.

2. Potts IF, Hirst E. Inverted papilloma of the bladder. J Urol. 1963;90:175-9.

3. Matz LR, Wishart VA, Goodman MA. Inverted urothelial papilloma Pathology. 1974;6:37-44.

4. Assor D. Inverted papilloma of the renal pelvis. J Urol. 1976;116:354.

5. Cameron MK, Lupton $\mathrm{CH}$. Inverted papilloma of the lower urinary tract. Br J Urol. 1976:48:567-77.

6. Di Cello V, Brischi G, Durval A, Mincione GP. Inverted papilloma of the ureteropelvic junction. J Urol. 1980;123:110.

7. Theoret G, Paquin F, Schick E, Martel A. Inverted papilloma of urinary tract. Urology. 1980;16:149-51.

8. Uyama T, Moriwaki S. Inverted papilloma with malignant change of renal pelvis. Urology. 1981;17:200-1.

9. Anderstrom C, Johansson S, Pettersson S. Inverted papilloma of the urinary tract. J Urol. 1982;127:1132-4.
10. Watters G, Grant A, Wiles S, Kneale K, Mitterdorfer A. Inverted papilloma of the upper urinary tract. Br J Urol. 1983:55:176-9.

11. Lausten GS, Anagnostaki L, OF T. Inverted papilloma of the upper urinary tract. Eur Urol. 1984;10:67-70.

12. Taylor FM, Arroyo JG. Inverted papilloma of the renal pelvis. Cytologic features of ureteral washings. Acta Cytol. 1986:30:166-8.

13. Schulze S, Holm-Nielsen A, Ravn V. Inverted papilloma of upper urinary tract. Urology. 1986;28:58-61.

14. Romanelli R. Inverted urothelial papilloma. Report of five cases and review of the literature. Pathologica. 1986;78:89-97.

15. Yamaguchi K, Kitagawa N, Zama S, Yanagi S, Ito H, Matsuzaki O, Nagao K. Inverted papilloma of renal pelvis associated with transitional cell carcinoma of the bladder. Urol Int. 1988:43:302-4.

16. Schultz RE, Boyle DE. Inverted papilloma of renal pelvis associated with contralateral ureteral malignancy and bladder recurrence. J Urol. 1988;139: 111-3.

17. Aubert J, Dore B, Villemonteix P, Touchard G. Inverted papilloma of upper urinary tract. Four case reports. Eur Urol. 1988;15:150-2.

18. Kyriakos M, Royce RK. Multiple simultaneous inverted papillomas of the upper urinary tract. A case report with a review of ureteral and renal pelvic inverted papillomas. Cancer. 1989;63:368-80.

19. Bagley DH, McCue P, Blackstone SA. Inverted papilloma of renal pelvis: flexible ureteroscopic diagnosis and treatment. Urology. 1990;36:336-8.

20. Bassi P, Piazza R, Milani C, Aragona F, Oliva G, Dalla PP. Inverted papilloma of the renal pelvis. Urol Int. 1991;46:73-6.

21. Vlassopoulos G, Sakkas G, Legaki S, Sofras F, Karagiannis A. Inverted papilloma of the renal pelvis. Int Urol Nephrol. 1992;24:345-6.

22. Ueda T, Akimoto S, Shimazaki J, Matsuzaki M, Nagao K. Inverted papilloma of the renal pelvis associated with renal cell carcinoma: a case report. Hinyokika Kiyo. 1992;38:561-3.

23. Spevack L, Herschorn S, Srigley J. Inverted papilloma of the urinary tract. J Urol. 1995:153:1202-4

24. Chiura AN, Wirtschafter A, Baglew DH. Upper urinary tract inverted papillomas. Urology. 1998;52:514-6.

25. Darras J, Inderadjaja N, Vossaert P. Synchronous inverted papilloma of bladder and renal pelvis. Urology. 2005:65:798.e25-8.

26. Luo JD, Wang P, Chen J, Liu B, Wang S, Shen BH, Xie LP. Upper urinary tract inverted papillomas: report of 10 cases. Oncol Lett. 2012:4:71-4.

27. Geisler $\mathrm{CH}$, Mori K, Leiter E. Lobulated inverted papilloma of the ureter. J Urol. 1980;123:270-1.

28. Silverstein SV, Carlton CE Jr. Inverted papilloma of ureter. Urology. 1981;17: 160-2.

29. Fromowitz FB, Steinbook ML, Lautin EM, Friedman AC, Kahan N, Bennett MJ, Koss LG. Inverted papilloma of the ureter. J Urol. 1981;126:113-6.

30. Ajrawat HS, Skogg DP, Asirwatham JE, Gonder MJ. Lobulated inverted papilloma of ureter. Urology. 1982;20:290-2.

31. Naito S, Minoda M, Hirata H. Inverted papilloma of ureter. Urology. 1983;22: 290-1.

32. Jacobellis U, Resta L, Ruotolo G. Inverted papilloma of the ureter. Eur Urol. 1983;9:370-1.

33. Embon OM, Saghi N, Bechar L. Inverted papilloma of ureter. Eur Urol. 1984; 10:139-40.

34. Perrin P, Dutrieux N, Durand L. Inverted papilloma of the ureter. Br J Urol. 1984;56:223

35. Mottola A, Selli C, Carini M. Inverted papilloma of the ureter. Ital J Surg Sci. 1984;14:341-4

36. Palvio DH. Inverted papillomas of the urinary tract. A case of multiple, recurring inverted papillomas of the renal pelvis, ureter and bladder associated with malignant change. Scand J Urol Nephrol. 1985;19:299-302.

37. Moss JG, Gunn AA. Inverted papilloma of the ureter lying within an ileal carcinoid. Br J Urol. 1987;60:272.

38. Corkill M, Srigley J, Graham R, Herschorn S. Inverted papilloma: an uncommon benign cause of a ureteral filling defect. Urol Radiol. 1987;9: 164-7.

39. Duchek M, Hallmans G, Hietala SO, Ljungberg B, Thore J. Inverted papilloma with intussusception of the ureter. Case report. Scand J Urol Nephrol. 1987; 21:147-9.

40. Abulafi A, Leese T, Osborn DE. Inverted papilloma of the ureter. Br J Urol. 1987;59:480

41. Villani U, Leoni S, Casolari E. Inverted papilloma of the ureter: two cases of conservative therapy. Eur Urol. 1987;13:125-7. 
42. Kostakopoulos A, Delakas D, Legaki S, Sofras F. Inverted papilloma of the ureter. Acta Urol Belg. 1988;56:471-4.

43. Garritano A, Vecchioli Scaldazza C, Morosetti C. Inverted papilloma of the ureter. Eur Urol. 1988;14:249-50.

44. Page CM, Nelson JH, Drago JR. Multifocal, synchronous inverted papillomas involving the ureter. J Urol. 1991;145:357-8.

45. Kunimi K, Uchibayashi T, Egawa M. A case of inverted papilloma of the ureter: is the DNA ploidy pattern associated with occurrence of transitional cell carcinoma of the bladder? Int Urol Nephrol. 1994;26:17-22.

46. de Knijff DW, Theunissen PH, Delaere KP. Inverted papilloma of the ureter with subsequent invasive bladder cancer. Acta Urol Belg. 1997;65:45-6.

47. Hoekx L, Wyndaele JJ. Bilateral ureteral inverted papilloma with synchronous transitional cell tumor of the bladder. Acta Urol Belg. 1998;66: 17-9.

48. Lyon MB, Zorn KC, Orvieto MA, Rapp DE, Gerber GS, Shalhav AL. Case report: laparoscopic resection of ureteral inverted papilloma. J Endourol. 2006:20:399-401

49. Kilciler M, Bedir S, Erdemir F, Ors O, Kibar Y, Dayanc M. Evaluation of urinary inverted papillomas: a report of 13 cases and literature review. Kaohsiung J Med Sci. 2008;24:25-30.

50. Mertziotis N, Kozyrakis D, Petrolekas A, Terzi M, Kapranos N. Inverted papilloma of the ureter: study of a rare case with emphasis on clinicopathologic implications. Can Urol Assoc J. 2012;6:e274-6.

51. Murtaza B, Akmal M, Niaz WA, Ahmad H, Mahmood A. Inverted papilloma of ureter: a rare cause of hydronephosis. J Coll Physicians Surg Pak. 2012;22: 542-4.

52. Lopez-Fontana G, Alvarez-Ossorio JL, Ramos Jl, Castineiras J, Moyano JL, Castillo OA. Ureteral inverted papilloma: laparoscopic distal ureterectomy and Boari flap. Arch Esp Urol. 2012;65:759-61.

53. Hartmann A, Dietmaier W, HofstadterF BLJ, Cheville JC, Blaszyk H. Urothelial carcinoma of the upper urinary tract: inverted growth pattern is predictive of microsatellite instability. Hum Pathol. 2003;34:222-7.

54. Sarode VR, Robinson L. Screening for lynch syndrome by immunohistochemistry of mismatch repair proteins. Significance of indeterminate result and correlation with mutational studies. Arch Pathol Lab Med. 2019;143:1225-33.

55. Jorgensen PH, Vainer B, Hermann GG. A clinical and molecular review of inverted papilloma of the urinary tract: how to handle? APMIS. 2015;123: 920-9.

56. Gupta R, Paner GP, Amin MB. Neoplasms of the upper urinary tract: a review with focus on urothelial carcinoma of the pelvicalyceal system and aspects related to its diagnosis and reporting. Adv Anat Pathol. 2008;15:127-39.

57. Amin M, Gomez J, Young R. Urothelial transitional cell carcinoma with endophytic growth patterns: a discussion of patterns of invasion and problems associated with assessment of invasion in 18 cases. Am J Surg Pathol. 1997;21:1057-68.

58. Wobker SE, Zhong M, Epstein Jl. Polypoid urothelial tumor with inverted growth pattern in the renal pelvis: morphologic and molecular characteristics of a unique diagnostic entity. Hum Pathol. 2017;59:26-33.

59. Jones TD, Zhang S, Lopez-Beltran A, Eble JN, Sung MT, MacLennan GT, Montironi R, Tan PH, Zheng S, Baldridge LA, Cheng L. Urothelial carcinoma with an inverted growth pattern can be distinguished from inverted papilloma by fluorescence in situ hybridization, immunohistochemistry, and morphologic analysis. Am J Surg Pathol. 2007;31:1861-7.

60. Cheville JC, Wu K, Sebo TJ, Cheng L, Riehle D, Lohse CM, Shane V. Inverted urothelial papilloma: is ploidy, MIB-1 proliferative activity, or p53 protein accumulation predictive of urothelial carcinoma. Cancer. 2000;88:632-6.

61. Broussard JN, Tan HP, Epstein J. Atypia in inverted urothelial papillomas: pathology and prognostic significance. Hum Pathol. 2004;351:499-504.

62. Eiber M, van Oers JM, Zwarthoff EC, van der Kwast TH, Ulrich O, Helpap B, Stoerkel S, Blaszyk H, Cheville J, Sauter G, Wild PJ, Stoehr R, Hofstaedter F, Hartmann A. Low frequency of molecular changes and tumor recurrence in inverted papillomas of the urinary tract. Am J Surg Pathol. 2007;31:938-46.

63. Harper HL, McKenney JK, Heald B, Stephenson A, Campbell SC, Plesec T, Magi-Galluzzi C. Upper tract urothelial carcinomas: frequency of association with mismatch repair protein loss and lynch syndrome. Mod Pathol. 2017; 30:146-56.

64. Giedl J, Schneckenpointner R, Filbeck T, Ruemmele P, Hofstaedter F, Burger M, Hartmann A, Stoehr R. Low frequency of HNPCC-associated microsatellite instability and aberrant MMR protein expression in early-onset bladder cancer. Am J Clin Pathol. 2014;142:634-9.
65. Picozzi S, Casellato S, Bozzini G, Ratti D, Macchi A, Rubino B, Pace G, Carmignani $L$. Inverted papilloma of the bladder: a review and an analysis of the recent literature of 365 patients. Urol Oncol. 2013;31:1584-90.

66. Patel P, Reikie BA, Maxwell JP, Yilmaz A, Gotto GT, Trpkov K. Long-term clinical outcome of inverted urothelial papilloma including cases with focal papillary pattern: is continuous surveillance necessary? Urology. 2013;82: 857-60.

\section{Publisher's Note}

Springer Nature remains neutral with regard to jurisdictional claims in published maps and institutional affiliations.
Ready to submit your research? Choose BMC and benefit from:

- fast, convenient online submission

- thorough peer review by experienced researchers in your field

- rapid publication on acceptance

- support for research data, including large and complex data types

- gold Open Access which fosters wider collaboration and increased citations

- maximum visibility for your research: over $100 \mathrm{M}$ website views per year

At BMC, research is always in progress.

Learn more biomedcentral.com/submissions 